Check for updates

Cite this: Chem. Commun., 2020, 56, 2127

Received 15th November 2019, Accepted 15th January 2020

DOI: $10.1039 / c 9 c c 08944 f$

rsc.li/chemcomm

\section{Pathway dependent shape-transformation of azide-decorated polymersomes $\dagger$}

\author{
Hailong Che, Lafayette N. J. de Windt, Jianzhi Zhu, Imke A. B. Pijpers, \\ Alexander F. Mason, Loai K. E. A. Abdelmohsen (D)* and Jan C. M. van Hest (D)*
}

\begin{abstract}
Here we report the shape transformation of poly(ethylene glycol)polystyrene (PEG-PS) polymersomes into ordered inverse morphologies, directed by the salt concentration of the medium and the presence of azide groups on the polymersome surface. The azide moieties introduced at the chain ends of the PEG blocks induce a difference in hydrodynamic volume of the hydrophilic domains at the inner and outer side of the vesicular membrane, allowing control over its spontaneous curvature and hence the pathway of shape deformation. This simple modification enables access to intricate morphologies which are traditionally only accessible via the application of complex polymer building blocks.
\end{abstract}

Among the most reported self-assembled polymeric nanoparticles, polymer vesicles, commonly referred to as polymersomes, have demonstrated morphological diversity due to their improved tailormade chemical design in comparison to their counterparts, liposomes, and their ability to be assembled under kinetic control. Furthermore, polymersome morphology transformations can be well-tuned by exploiting external chemical or physical stimuli (i.e., osmosis, $\mathrm{pH}$, light, temperature, redox, or gas) ${ }^{1-10}$ which is useful for the design and construction of adaptive artificial biomimetic systems. Well-defined polymersomes with morphology control also have biomedical relevance. ${ }^{11,12}$ For example, it has been shown that the cellular uptake efficiency of nanoparticles is highly dependent on the particle shape, which influences the rate and pathway of cellular internalization. ${ }^{13}$ In addition, bowl-shaped polymersomes, such as stomatocytes show many exciting opportunities as nanomotors and nanoreactors. ${ }^{14,15}$

Among the range of polymer assemblies available, block copolymer (BCP)-based ordered inverse morphologies have gained much research interest owing to their high internal surface area and accessibility for a wide range of molecules,

Eindhoven University of Technology, Institute for Complex Molecular Systems,

P.O. Box 513 (STO 3.41), 5600MB Eindhoven, The Netherlands.

E-mail: j.c.m.v.hest@tue.nl, l.k.e.a.abdelmohsen@tue.nl

$\dagger$ Electronic supplementary information (ESI) available: Synthesis, NMR, GPC, TEM and DLS results. See DOI: 10.1039/c9cc08944f which enable their use in biomedical science as controlled delivery vehicles, and as templates for the preparation of nanomaterials. ${ }^{16-18}$ More than 20 years ago, the Eisenberg group was the first to report mesosized crystal-like aggregates with an internal structure of hexagonally packed hollow hoops (HHHs) by self-assembly of polystyrene- $b$-poly(acrylic acid) (PS- $b$-PAA). ${ }^{19}$ More recently, Kim and co-workers developed cubosomes and hexasomes with inverse bicontinuous cubic structures by dendritic-linear BCPs bearing a dendritic hydrophilic block. ${ }^{20-22}$ Additionally, polymerization-induced self-assembly (PISA) was found to be a facile strategy to generate such inverse morphologies. ${ }^{23,24}$ Despite these significant achievements, most reported inverse morphology preparation methods require complex synthesis of the building blocks and strict preparation conditions, which restricts their utility. Thus, it is of interest to create well-defined polymeric inverse morphologies via a facile, easily applicable strategy.

Previously our group reported the formation of bowl-shaped stomatocytes via the osmotically triggered shape transformation of spherical poly(ethylene glycol)-b-poly(styrene) (PEG-b-PS) polymersomes. ${ }^{1}$ To enhance their utility in nanomedicine, we have recently shown the shape transformation of poly(ethylene glycol)-block-poly(D,L-lactide) (PEG- $b$-PDLLA) into biodegradable nanotubes and stomatocytes, also with the help of the dialysis methodology. ${ }^{25,26}$ In order to further explore the osmotically induced shape transformation of polymersomes and gain more control over such deformations, here we present an unusual morphology transition from polymersomes to hexagonally packed hollow hoops (HHHs), mediated by the presence of azide groups on the polymersome surface (Scheme 1).

Two types of block copolymers $\mathrm{mPEG}_{45}-b-\mathrm{PS}_{205}$ and $\mathrm{N}_{3}-\mathrm{PEG}_{45^{-}}$ $b$ - $\mathrm{PS}_{200}$ were prepared by atom transfer radical polymerization (ATRP) (details of polymer synthesis and characterization are given in the ESI $\dagger$ ). The self-assembly of these polymers was performed using a nanoprecipitation method, whereby deionized water was added dropwise to a copolymer solution in an organic solvent ( $4: 1 \mathrm{THF} /$ dioxane) up to $50 \mathrm{vol} \%$, producing a cloudy suspension. The produced suspension was dialyzed against pure water or aqueous solutions with varying salt $(\mathrm{NaCl})$ 

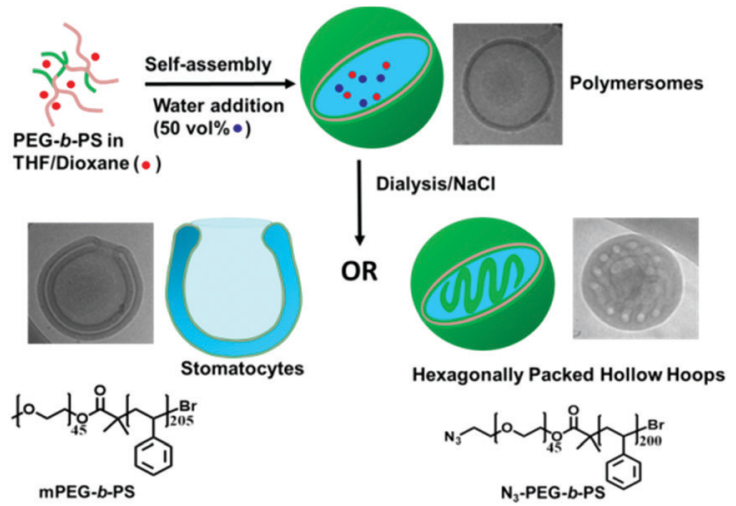

Scheme 1 Schematic illustration of the pathway dependent shapetransformation of polymersomes mediated by salt and azide groups. During the process of dialysis against $\mathrm{NaCl}, \mathrm{mPEG}_{45}-b-\mathrm{PS}_{205}$ polymersomes transform into stomatocytes, while $\mathrm{N}_{3}-\mathrm{PEG}_{45}-b-\mathrm{PS}_{200}$ polymersomes shape change into internally structured $\mathrm{HHHs}$

concentrations $(5,10,25$, and $50 \mathrm{mM})$ at room temperature. After overnight dialysis against pure water, $\mathrm{mPEG}_{45}-b-\mathrm{PS}_{205}$ yielded well-defined vesicular polymersomes, similar to rapidly quenched polymersomes, which was confirmed by transmission electron microscopy (TEM) (Fig. S4a and S5, ESI $\dagger$ ). After dialysis of the suspension against a $\mathrm{NaCl}$ solution, the average hydrodynamic radius $\left(R_{\mathrm{h}}\right)$ of the assemblies, as determined by dynamic light scattering (DLS), demonstrated a slight decrease compared to the samples dialyzed against water (Fig. S6, ESI $†$ ). TEM demonstrated that the morphology of the polymersomes completely changed from spheres to bowl-shaped stomatocytes, which was further confirmed by scanning electron microscopy (SEM) and cryo-TEM (Fig. S4b-d, ESI $\dagger$ ). Moreover, with an increase in salt concentration, the average opening size of the stomatocytes witnessed a continuous decrease from an original value of $245 \pm 50 \mathrm{~nm}(5 \mathrm{mM} \mathrm{NaCl})$ to $170 \pm 20 \mathrm{~nm}(10 \mathrm{mM} \mathrm{NaCl}), 90 \pm 15 \mathrm{~nm}(25 \mathrm{mM} \mathrm{NaCl})$, and $55 \pm 10 \mathrm{~nm}(50 \mathrm{mM} \mathrm{NaCl})$ (Fig. S7, ESI $\dagger$ ), which is in accordance with our previous work. ${ }^{26}$

In the case of $\mathrm{N}_{3}-\mathrm{PEG}_{45}-b-\mathrm{PS}_{200}$ assemblies, polymersomes were obtained after dialysis against pure water, indicating that the azide groups do not have an influence on the morphology in the absence of salt (Fig. 1a). However, when the same dialysis method against $\mathrm{NaCl}$ was applied to $\mathrm{N}_{3}-\mathrm{PEG}_{45}-b$ - $\mathrm{PS}_{200}$ polymersomes, to our surprise unusual self-assemblies were formed, which were very similar to the previously reported HHHs by the Eisenberg group. ${ }^{19}$ As shown in Fig. $1 \mathrm{~b}$ and Fig. S8 (ESI $\dagger$ ), the dark regions with a width of $20-30 \mathrm{~nm}$ correspond to the areas where block copolymer is present, while the bright regions with a diameter of $30-40 \mathrm{~nm}$ represent polymer-devoid domains, which is similar to previously reported results. ${ }^{19}$ These nanoparticles with inverse mesophases were confirmed by SEM, from which we can clearly see that these particles comprise patterned surfaces (Fig. 1c). The special swirling patterns are proposed to be generated because of the alignment of the bicontinuous channels, which are formed between neighboring channels during the process of dialysis. Compared to traditional spherical polymeric particles with smooth surfaces, the distinct a)

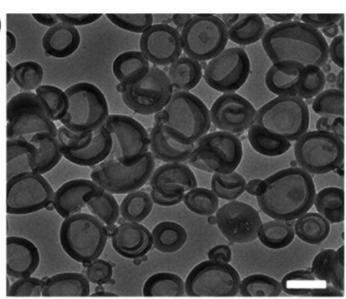

c)

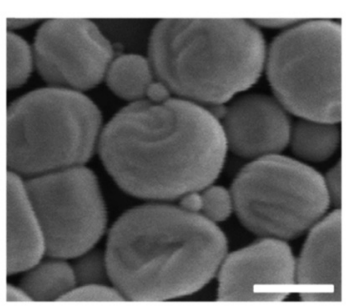

b)

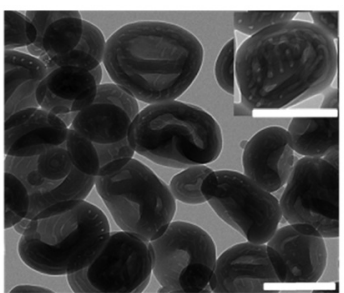

d)

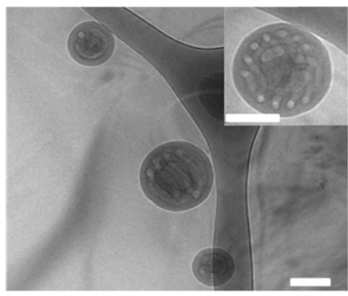

Fig. 1 TEM images of $\mathrm{N}_{3}-\mathrm{PEG}_{45}-b-\mathrm{PS}_{200}$ (a) polymersomes and (b) $\mathrm{HHHs}$. (c) SEM and (d) cryo-TEM images of $\mathrm{N}_{3}-\mathrm{PEG}_{45}-b-\mathrm{PS}_{200} \mathrm{HHHs}$. The HHHs were prepared by dialysis of an $\mathrm{N}_{3}-\mathrm{PEG}_{45}-b-\mathrm{PS}_{200}$ suspension against $50 \mathrm{mM} \mathrm{NaCl}$ solution. All scale bars are $200 \mathrm{~nm}$.

swirling patterns on the surface of these particles underscore their organized internal structures, which was confirmed by cryo-TEM (Fig. 1d).

To further study how the azide-functionalized polymersomes transformed into HHHs, the self-assembled $\mathrm{N}_{3}-\mathrm{PEG}_{45^{-}}$ $b$ - PS $_{200}$ polymersomes were dialyzed against aqueous solutions with varying salt concentrations $(5,10,25$ and $50 \mathrm{mM})$. It was observed that after dialysis against $5 \mathrm{mM} \mathrm{NaCl}$, the polymersomes transformed into stomatocytes, while when higher salt concentrations ( $10 \mathrm{mM}$ and $25 \mathrm{mM}$ ) were applied, the stomatocytes started transforming into HHHs (Fig. 2), demonstrating that stomatocytes are the intermediate morphologies, which can further evolve into HHHs by increasing salt concentrations.

The above results indicate that $\mathrm{N}_{3}-\mathrm{PEG}_{45}-b-\mathrm{PS}_{200}$ forms particles with an internal structure in the presence of salt. To characterize these particles further, we performed light scattering measurements on dilute solutions of $\mathrm{N}_{3}-\mathrm{PEG}_{45}-b$ - $\mathrm{PS}_{200}$ dialyzed against $50 \mathrm{mM} \mathrm{NaCl}$ solution (Fig. S9, ESI $\dagger$ ). These samples were studied at multiple angles to determine the hydrodynamic radius and the radius of gyration $\left(R_{\mathrm{g}}\right.$, details of analysis are given in the ESI $\dagger$ ). Interestingly, we found that the values of $R_{g}$ were an order of magnitude smaller than the values of $R_{\mathrm{h}}$ (Table $\mathrm{S} 1$, ESI $\dagger$ ). This finding suggests that the mass of the particles is distributed over not only an outer surface but also an internal structure, which further supports the formation of HHHs. Moreover, the average diameter of polymersomes and HHHs was reduced after dialysis, while the distribution of diameter didn't change, which shows that no fusion happens during the shape transformation (Fig. S10 and Table S2, ESI $\dagger$ ).

Next we sought to explore whether it is the presence of azide groups that induces the deformation of polymersomes. For this purpose, the $\mathrm{N}_{3}-\mathrm{PEG}_{45}-b-\mathrm{PS}_{200}$ based assemblies were first reacted with dibenzocyclooctyne-PEG 4 -alcohol (DBCO-PEG 4 - $-\mathrm{OH}$ ) to make sure that there were no azide groups exposed on the surface of the assemblies. After overnight reaction, the mixture was dialyzed against pure water or salt solution. Interestingly, no shape-transformation 

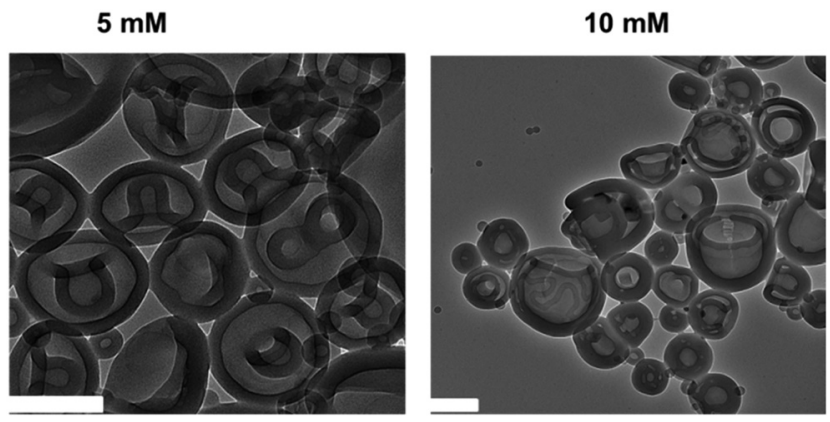

$25 \mathrm{mM}$

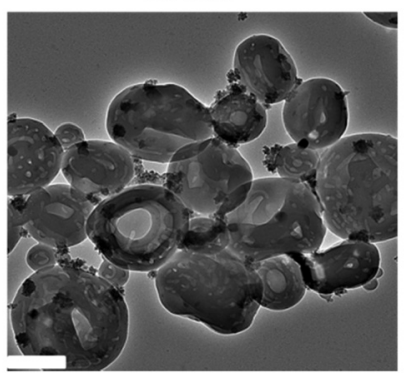

Fig. 2 TEM images of $\mathrm{N}_{3}-\mathrm{PEG}_{45}-b-\mathrm{PS}_{200}$ after dialysis against $5 \mathrm{mM}, 10 \mathrm{mM}$, and $25 \mathrm{mM} \mathrm{NaCl}$, respectively. All scale bars are $200 \mathrm{~nm}$.

was observed in either case (Fig. S11, ESI $\dagger$ ). These results suggest that the azide groups play a key role in the morphology transition from polymersomes to HHHs, in the presence of salt.

Having successfully accomplished a pathway dependent shapetransformation of polymersomes mediated by salt and azide groups, the reversibility of the method was studied. Our previous work showed that reverse dialysis allows PEG-PDLLA stomatocytes to regain their original spherical polymersome structure. ${ }^{27}$ Here we performed a similar procedure on deformed $\mathrm{MPEG}_{45}-b-\mathrm{PS}_{205}$ stomatocytes and $\mathrm{N}_{3}-\mathrm{PEG}_{45}-b$ - $\mathrm{PS}_{200} \mathrm{HHHs}$, which were dialyzed against $50 \mathrm{mM} \mathrm{NaCl}$ solution mixed with $50 \mathrm{vol} \%$ organic solvent (4:1 THF/dioxane) at room temperature. After $1 \mathrm{~h}$ dialysis, the morphologies of the particles were characterized by TEM. In the case of $\mathrm{mPEG}_{45}-b$ - $\mathrm{PS}_{205}$ stomatocytes the reverse dialysis resulted in recovery of spherical polymersomes since organic solvent can replasticize the PS membrane, and thus the assemblies tend to form the energetically favoured spherical state (Fig. S12, ESI $\dagger$ ). However, in the case of $\mathrm{N}_{3}-\mathrm{PEG}_{45}-b-\mathrm{PS}_{200} \mathrm{HHHs}$, the results were more complex. As shown in Fig. 3, after reverse dialysis, the $\mathrm{N}_{3}-\mathrm{PEG}_{45}-b-\mathrm{PS}_{200} \mathrm{HHHs}$ transformed into porous-like structures other than spherical vesicular polymersomes. The surface morphology of these self-assemblies was further probed using SEM to reveal that the patches on the particle surface were nanopores, as shown in Fig. 3b. One possible reason for the formation of these porous structures is that the internal bicontinuous domains become swollen in the presence of an organic solvent, which as a result fold outwards into the observed topology.

Having confirmed the reproducibility and reversibility of the above shape transformation process, we sought to understand the mechanism on how salt and azide groups act as driving forces to direct the morphology transformation of the polymersomes. According to eqn (1), the spontaneous curvature $\left(C_{0}\right)$ of

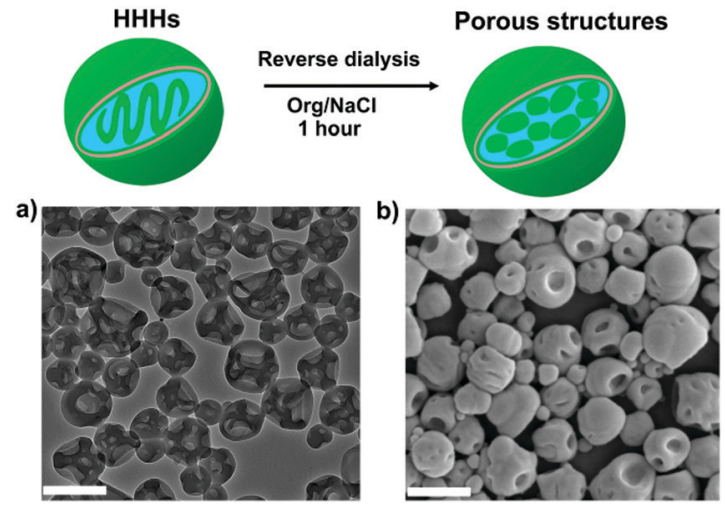

Fig. 3 Schematic illustration of the reverse dialysis process of $\mathrm{N}_{3}-\mathrm{PEG}_{45^{-}}$ $b-\mathrm{PS}_{200} \mathrm{HHHs}$. (a) TEM and (b) SEM images of the porous structures after dialysis against 50 vol\% organic solvent. Scale bars are $500 \mathrm{~nm}$.

the BCP membrane plays a critical role in directing the deformation process.

$$
E_{\mathrm{b}}=\frac{k}{2} \oint\left(2 C-C_{0}\right)^{2} \mathrm{~d} A
$$

where $E_{\mathrm{b}}$ is the bending energy of the membrane, $k$ is the bending rigidity of the membrane, $C$ is the mean surface curvature, $C_{0}$ is the spontaneous curvature and $A$ is the surface area. Generally, spherical polymersomes undergo a reduction in volume during the process of dialysis against salt, and the effect of osmotic pressure can be orders of magnitude greater than $E_{\mathrm{b}}$. Based on existing theory, spherical polymersomes tend to transform into oblates (toward discs and stomatocytes) if the contribution of $C_{0}$ is enough during the shape transformation process. $^{28-30}$ The dialysis process causes quick outflow of organic solvent and water, giving rise to internal volume reduction and transient changes in PEG conformation. The rate of the outflow of water can be enhanced by increasing the ionic strength of the dialysis medium-leading to a higher organic solvent to water ratio inside the polymersomes. Such rate enhancement accentuates the PEG anisotropy between the inner and outer surfaces, as the PEG inner layer is more swollen and hence occupies a larger hydrodynamic volume. This leads to a larger contribution of a negative $C_{0}$ during the shape transformation process. This is the process by which the spherical polymersomes shape transform into stomatocytes. In the case of azide polymersomes, we expect that the membrane anisotropy is further enhanced by the formation of solvation shells at the PEG chain end as a result of azide coordination to water molecules. ${ }^{31}$ In comparison to the outer volume $(1000 \mathrm{~mL})$, the inner polymersome volume is very small and therefore, coordination of water molecules to the azide moieties intensifies the solvation effect of the organic solvent to PEG, leading to an increased hydrodynamic volume of the PEG chains at the internal side of the vesicles, compared to the hydrodynamic volume these hydrophilic chains occupy at the external side. This anisotropy $\left(V_{\mathrm{h}}(\mathrm{int})>V_{\mathrm{h}}(\mathrm{ext})\right)$ results in a larger contribution of $C_{0}$ to the shape transformation process. The difference in hydrodynamic chain volume thus acts as a steric driving force 
for directing the shape transformation via the oblate pathway toward stomatocytes and further towards the formation of HHHs.

In conclusion, we have presented an unusual shapetransformation of PEG-PS polymersomes mediated by salt and azide groups present at the polymersome surface. Copolymers with narrow dispersity index were utilized to prevent any kind of domain formation that would impact the shape transformation process. Traditional $\mathrm{mPEG}_{45}-b-\mathrm{PS}_{205}$ polymersomes deform into stomatocytes induced by osmotic pressure during the process of dialysis against salt solutions, while azide-modified $\mathrm{N}_{3}-\mathrm{PEG}_{45}-b$ $\mathrm{PS}_{200}$ undergo a transition from polymersomes to high internally organized HHHs. Importantly, the formation of stomatocytes and HHHs could be finely tuned by the concentration of salt. This new approach towards the formation of HHHs provides an interesting additional method for the development of polymeric nanoparticles with sophisticated internal structure. The detailed underlying mechanism of the reported polymeric inverse morphology is topic of further investigation.

The authors acknowledge the Dutch Science Foundation (VICI grant), the ERC Advanced grant Artisym 694120, the Dutch Ministry of Education, Culture and Science (Gravitation program 024.001.035) and the European Union's Horizon 2020 research and innovation programme Marie Sklodowska Curie Innovative Training Network Nanomed, (No. 676137) for funding.

\section{Conflicts of interest}

There are no conflicts to declare.

\section{Notes and references}

1 K. T. Kim, J. Zhu, S. A. Meeuwissen, J. J. Cornelissen, D. J. Pochan, R. J. M. Nolte and J. C. M. van Hest, J. Am. Chem. Soc., 2010, 132, 12522-12524.

2 J. Du and S. P. Armes, J. Am. Chem. Soc., 2005, 127, 12800-12801.

3 J. Rodríguez-Hernández and S. Lecommandoux, J. Am. Chem. Soc., 2005, 127, 2026-2027.

4 C. Pietsch, U. Mansfeld, C. Guerrero-Sanchez, S. Hoeppener, A. Vollrath, M. Wagner, R. Hoogenboom, S. Saubern, S. H. Thang and C. R. Becer, Macromolecules, 2012, 45, 9292-9302.

5 Y. Li, B. S. Lokitz and C. L. McCormick, Angew. Chem., Int. Ed., 2006, 45, 5792-5795.
6 Q. Yan, J. Yuan, Z. Cai, Y. Xin, Y. Kang and Y. Yin, J. Am. Chem. Soc., 2010, 132, 9268-9270.

7 Q. Yan and Y. Zhao, Angew. Chem., Int. Ed., 2013, 52, 9948-9951.

8 H. Che and J. Yuan, Macromol. Res., 2017, 25, 635-639.

9 M. C. M. van Oers, F. P. J. T. Rutjes and J. C. M. van Hest, J. Am. Chem. Soc., 2013, 135, 16308-16311.

10 C. K. Wong, M. H. Stenzel and P. Thordarson, Chem. Soc. Rev., 2019, 48, 4019-4035.

11 D. S. Williams, I. A. B. Pijpers, R. Ridolfo and J. C. M. van Hest, J. Controlled Release, 2017, 259, 29-39.

12 I. A. B. Pijpers, L. K. E. A. Abdelmohsen, Y. Xia, S. Cao, D. S. Williams, F. Meng, J. C. M. van Hest and Z. Zhong, Adv. Ther., 2018, 1, 1800068.

13 S. E. A. Gratton, P. A. Ropp, P. D. Pohlhaus, J. C. Luft, V. J. Madden, M. E. Napier and J. M. DeSimone, Proc. Natl. Acad. Sci. U. S. A., 2008, 105, 11613.

14 D. A. Wilson, R. J. M. Nolte and J. C. M. van Hest, Nat. Chem., 2012, 4, 268.

15 M. Nijemeisland, L. K. E. A. Abdelmohsen, W. T. S. Huck, D. A. Wilson and J. C. M. van Hest, ACS Cent. Sci., 2016, 2, 843-849.

16 H. He, K. Rahimi, M. Zhong, A. Mourran, D. R. Luebke, H. B. Nulwala, M. Möller and K. Matyjaszewski, Nat. Commun., 2017, 8, 14057.

17 D. Yang, D. F. O'Brien and S. R. Marder, J. Am. Chem. Soc., 2002, 124, 13388-13389.

18 A. Angelova, B. Angelov, B. Papahadjopoulos-Sternberg, M. Ollivon and C. Bourgaux, Langmuir, 2005, 21, 4138-4143.

19 L. Zhang, C. Bartels, Y. Yu, H. Shen and A. Eisenberg, Phys. Rev. Lett., 1997, 79, 5034-5037.

20 Y. La, C. Park, T. J. Shin, S. H. Joo, S. Kang and K. T. Kim, Nat. Chem., 2014, 6, 534.

21 T. H. An, Y. La, A. Cho, M. G. Jeong, T. J. Shin, C. Park and K. T. Kim, ACS Nano, 2015, 9, 3084-3096.

22 C. Park, Y. La, T. H. An, H. Y. Jeong, S. Kang, S. H. Joo, H. Ahn, T. J. Shin and K. T. Kim, Nat. Commun., 2015, 6, 6392.

23 W. J. Zhang, C. Y. Hong and C. Y. Pan, Macromol. Rapid Commun., 2015, 36, 1428-1436.

24 F. Lv, Z. An and P. Wu, Nat. Commun., 2019, 10, 1397.

25 L. K. E. A. Abdelmohsen, D. S. Williams, J. Pille, S. G. Ozel, R. S. M. Rikken, D. A. Wilson and J. C. M. van Hest, J. Am. Chem. Soc., 2016, 138, 9353-9356.

26 I. A. Pijpers, L. K. E. A. Abdelmohsen, D. S. Williams and J. C. M. van Hest, ACS Macro Lett., 2017, 6, 1217-1222.

27 S. A. Meeuwissen, K. T. Kim, Y. Chen, D. J. Pochan and J. C. M. van Hest, Angew. Chem., Int. Ed., 2011, 50, 7070-7073.

28 C. K. Wong, A. F. Mason, M. H. Stenzel and P. Thordarson, Nat. Commun., 2017, 8, 1240.

29 R. Lipowsky, Faraday Discuss., 2013, 161, 305-331.

30 U. Seifert, K. Berndl and R. Lipowsky, Phys. Rev. A: At., Mol., Opt. Phys., 1991, 44, 1182-1202.

31 X. Yang, B. Kiran, X.-B. Wang, L.-S. Wang, M. Mucha and P. Jungwirth, J. Phys. Chem. A, 2004, 108, 7820-7826. 